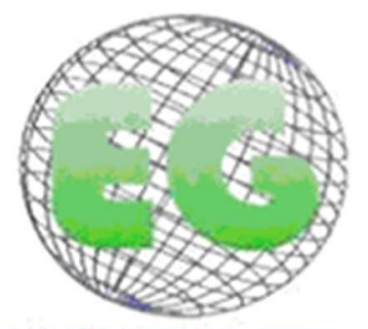

ISSN 1695-6141 $\mathbf{N}^{\circ} 63$

\title{
ORIGINALES
}

\section{Dinámicas de información en profesionales de enfermería desde el análisis de redes sociales}

Information dynamics in nursing professionals based on social network análisis

\author{
María-Lara Martínez-Gimeno ${ }^{1,2}$ \\ María-Antonia Ovalle-Perandones ${ }^{2,3}$ \\ Gema Escobar-Aguilar ${ }^{1}$ \\ Nélida Fernández-Martínez ${ }^{4}$ \\ José Alberto Benítez-Andrades ${ }^{2,5}$ \\ Pilar Marques-Sánchez ${ }^{2}$
}

1 Fundación San Juan de Dios. Centro Universitario de Ciencias de la Salud San Rafael-Nebrija. Universidad Nebrija. Madrid. España. gescobar@nebrija.es

2 Grupo de Investigación SALBIS. Facultad de Ciencias de la Salud, Universidad de León, Campus, Ponferrada. España.

3 Facultad de Ciencias de la Documentación. Departamento de Biblioteconomía y Documentación. Universidad Complutense de Madrid. Madrid. España.

4 Departamento de Ciencias Biomédicas. Facultad de Veterinaria. Universidad de León. Campus de Vegazana, León, España.

5 Departamento de Ingeniería Eléctrica y de Sistemas y Automática. Universidad de León, Campus de Vegazana, León, España.

\section{https://doi.org/10.6018/eglobal.470721}

Recibido: 26/02/2021

Aceptado: $3 / 03 / 2021$

\section{RESUMEN:}

Introducción: El conocimiento es una herramienta necesaria para la investigación científica y el progreso de cualquier disciplina. Pero el conocimiento científico y las dinámicas de información no sólo están sostenidas por los individuos, sino que son producidas y mantenidas por grupos de personas que trabajan en un mismo entorno donde los vínculos y las relaciones pueden influir en el proceso.

Objetivo: Analizar las redes sociales de utilización de fuentes de información, de ayuda/consejo para la transferencia de conocimiento y los lugares donde los profesionales de enfermería comparten información.

Método: Análisis de Redes Sociales a través de un cuestionario validado. Se reclutaron profesionales de 6 unidades hospitalarias.

Resultados: Participaron 77 profesionales con una edad media de 42,9 (DE:11,48). Los compañeros son la fuente de información más utilizada (76 elecciones) frente a las bases de datos y artículos científicos que son la menos seleccionada (63 elecciones). Las redes homófilas horizontales (profesionales con estatus/intereses similares) son las más frecuentes para obtener información sobre resultados de investigación (74 elecciones). La unidad asistencial es el entorno más señalado para compartir información (50 elecciones).

Conclusiones: Los profesionales consideran el conocimiento de sus compañeros como la principal fuente para obtener información sobre resultados de investigación. Unidades con determinado grado de 
especialización utilizan guías de práctica clínica y protocolos como fuente principal de información. Los profesionales de enfermería utilizan redes homófilas-horizontales para obtener información. El entorno laboral en sus diferentes ámbitos (unidad, office, reuniones) es el más utilizado para compartir información sobre resultados de investigación.

Palabras clave: Análisis de Redes Sociales; Fuentes de información; Gestión del conocimiento; Enfermería; Práctica basada en la evidencia; Utilización de la investigación

\begin{abstract}
:
Introduction: Knowledge is a necessary tool for scientific research and progress in any discipline. But scientific knowledge and information dynamics are not only sustained by individuals but are produced and maintained by groups of people working in the same environment where links and relationships can influence the process.

Aim: To analyze the social networks of information source utilization, help/advice for knowledge transfer and the places where nursing professionals share information.

Method: Analysis of social networks through a validated questionnaire. Professionals from 6 hospital units were recruited.

Results: 77 professionals participated with a mean age of 42.9 (SD:11.48). Peers were the most frequently used source of information (76 choices) compared to databases and scientific articles, which were the least selected (63 choices). Horizontal homophilous networks (professionals with similar status/interests) are the most frequent for obtaining information on research results (74 choices). The care unit is the most pointed environment for sharing information (50 choices).

Conclusions: Professionals consider the knowledge of their peers as the main source for obtaining information on research results. Units with a certain degree of specialization use clinical practice guidelines and protocols as the main source of information. Nursing professionals use homophilichorizontal networks to obtain information. The work environment in its different settings (unit, office, meetings) is the most used for sharing information on research results.
\end{abstract}

Key words: Social Network Analysis; Information sources; Knowledge Management; Nursing; Evidence-Based Practice; Research utilization.

\title{
INTRODUCCIÓN
}

El conocimiento, condicionado por las dinámicas de información, es una herramienta necesaria para la investigación científica y el progreso de cualquier disciplina ${ }^{(1)}$. El conocimiento tácito se relaciona con los elementos basados en la experiencia que son utilizados para la resolución de problemas y el conocimiento explícito hace referencia a la información formal obtenida mediante la literatura científica y documentos especializados; como son las guías de práctica clínica, los protocolos de actuación estandarizados, las revistas científicas y los libros, entre otros ${ }^{(2)}$.

La subestimación del conocimiento tácito, junto con la complejidad del contexto asistencial de la práctica, se interrelacionan como posibles causas de la brecha existente entre los resultados de la investigación y la toma de decisiones clínicas. Esta situación se visibiliza en el rechazo de algunos profesionales para modificar su práctica cuando deben incorporar aquellas evidencias disponibles que no guardan relación con su experiencia. Sin embargo, el conocimiento tácito adquiere un gran reconocimiento cuando proviene de profesionales con experiencia(2). En muchas ocasiones, los profesionales de enfermería recurren a la experiencia de otros profesionales para informar su práctica y esa experiencia puede estar, o no, sustentada en la investigación ${ }^{(3)}$.

El conocimiento efectivo probablemente dependa de la combinación de una base de conocimiento tácito, obtenido a través de la práctica clínica, con el conocimiento científico o explícito. Por ello, las fuentes de información para la toma de decisiones 
en el marco de la enfermería, incluyen tanto conocimiento tácito como explícito(3). Esta realidad permite afirmar que ni el conocimiento más explícito está carente de conocimiento tácito; ya que la utilización del mismo se realiza en un contexto determinado, vinculado a unos principios éticos y morales, la propia cultura y las vivencias personales del individuo ${ }^{(2)}$.

Por lo tanto, la utilización de la investigación puede entenderse como un proceso en el que los profesionales de la salud combinan el conocimiento explícito, basado en la investigación, con el conocimiento tácito basado en la práctica; es decir, ambos conocimientos se unen para ser utilizados en el contexto de su trabajo diario ${ }^{(4)}$.

Por otro lado, desde hace más de 4 décadas los estudios establecen la existencia de una gran separación entre la investigación y la práctica clínica, lo que ha supuesto que los resultados de la investigación no contribuyan a la mejora asistencial, como en un principio sería deseable ${ }^{(5)}$. Este hecho tiene lugar en un contexto que implica directamente al profesional de enfermería con la utilización de la investigación. Primero porque los profesionales de enfermería cada vez tienen una mayor conciencia y accesibilidad a la evidencia, y segundo porque el rol enfermero está adquiriendo unas dimensiones nunca vistas donde la investigación juega un papel trascendental. Prueba de ello es la designación por parte de la OMS y el CIE del año 2020 como el año Internacional de la Enfermería que ha dado lugar al movimiento Nursing Now; poniendo el foco en la posición trascendental que ocupa la enfermería en los diferentes escenarios de salud y en la responsabilidad que esta adquiere para generar evidencia sólida que pueda demostrar el impacto de los cuidados en la salud de los ciudadanos, las personas, la comunidad, entre otros ${ }^{(6)}$.

Pero para generar evidencia y analizar su impacto es importante comprender cómo los profesionales de enfermería gestionan el conocimiento científico. Así mismo, hay que tener en cuenta que el conocimiento científico no sólo es sostenido por los individuos, sino que es producido y mantenido por grupos de personas que trabajan en un mismo entorno, donde los vínculos y las relaciones entre los profesionales pueden influir en el proceso(4).

Parcham et al. informan que dentro del ámbito sanitario los distintos grupos profesionales establecen diferentes redes sociales; señalando cómo los médicos, por ejemplo, tienden a crear redes informales horizontales, relacionándose entre profesionales con estatus e intereses similares; es el denominado efecto de la homofilia, para así obtener información de otros médicos con más experiencia en su especialidad. Por otro lado, reflejan que los profesionales de enfermería suelen establecer redes verticales formales, relacionándose con profesionales que tienen un estatus e intereses diferentes ${ }^{(7)}$. Las redes horizontales son más efectivas para hacer uso de la influencia de los compañeros, mientras que las redes verticales son más efectivas para la aprobación de decisiones ${ }^{(8)}$.

Los estudios que han explorado cómo el conocimiento científico se identifica, circula, evalúa y utiliza en las organizaciones sanitarias, sugieren que antes de que éste pueda contribuir a iniciativas de cambio organizacional, debe ser incorporado en el conocimiento propio construido y compartido por las personas que componen la organización. Luego el conocimiento depende de su circulación en las redes interpersonales, y se diseminará si se tienen en cuenta esos vínculos sociales y se identifican y superan las barreras que puedan existir ${ }^{(9)}$. 
Para captar las relaciones o redes profesionales que coexisten en las organizaciones sanitarias, se dispone de una metodología que permite identificar las estructuras de las relaciones que se generan durante la utilización de la información para influir en el conocimiento: el Análisis de Redes Sociales (ARS). El ARS se basa en teorías y técnicas para comprender cómo las relaciones sociales influyen en los comportamientos ${ }^{(10)}$.

El uso del ARS está cada vez más presente en el contexto sanitario y su aplicabilidad es amplia. Focalizado en el ámbito de la educación para la salud se han publicado estudios que se centran en aspectos relacionados con la adquisición de estilos de vida saludables en el contexto de la interacción entre iguales. Un estudio realizado en el ámbito escolar con adolescentes exploró la relación entre el estatus socioeconómico y la obesidad, analizando los patrones de contacto entre pares teniendo en cuenta este parámetro(11). En esa misma línea, el estudio realizado por Quiroga et al. se focalizó en las redes de amistad de los adolescentes y el consumo de alcohol, para dar soporte al diseño de estrategias preventivas teniendo en cuenta cómo determinadas variables (nivel socioeconómico, sobrepeso, consumo de alcohol, patrón de amistad) influyen en la formación de redes sociales que median en la adquisición de estilos de vida saludables ${ }^{(12)}$.

En el contexto hospitalario, un estudio realizado en el ámbito de los cuidados paliativos utilizó el ARS para explorar cómo se desarrolla el proceso de comunicación entre profesionales de enfermería, paciente y entorno. Lo que permitió comprender determinas conductas y planificar estrategias para su mejora ${ }^{(13)}$. O estudios como el realizado por Creswick \& Westbrook en el que exploraron las redes de consejo de profesionales de enfermería respecto a la utilización de medicamentos ${ }^{(14)}$.

Por lo tanto, si partimos de que es un hecho demostrado que los profesionales de la salud influyen en el proceso de implantación del conocimiento científico en la práctica clínica, al formar parte de subculturas profesionales que intervienen en la difusión de ideas y conocimientos ${ }^{(15)}$, el ARS puede ayudar a entender determinados fenómenos relacionados con la difusión y utilización de la evidencia en profesionales de enfermería, que tienen que ver con las relaciones interpersonales; y con ello, analizar el contexto en el que se dan esas conexiones y dinámicas para la obtención de información.

El objetivo de esta propuesta es analizar las redes sociales de utilización de fuentes de información, de ayuda o consejo para la transferencia de conocimiento y de los lugares donde los profesionales de enfermería comparten información.

\section{MATERIAL Y MÉTODOS}

El ámbito de estudio es el Hospital Universitario de Móstoles (Comunidad Autónoma de Madrid) durante el año 2018. Este centro hospitalario participó en el proyecto de implantación de evidencia científica Sumamos Excelencia ${ }^{\circledR}$, siendo el centro con más unidades participantes ${ }^{(16)}$.

Los sujetos de estudio son profesionales de enfermería (TCAE/DUE) pertenecientes a seis unidades de ese hospital. Cinco de ellas habían participado seis meses antes en el proyecto antes descrito, no así la sexta (considerada unidad de control). 
Los criterios de inclusión de los profesionales de enfermería de las unidades participantes eran profesionales fijos en plantilla relacionados con el proceso asistencial que habían formado parte del proyecto de implantación Sumamos Excelencia ${ }^{\circledR}$; profesionales gestores que participaron en el proyecto de implantación y profesionales que aceptaran participar en el estudio. Quedaron excluidos los profesionales con contratos temporales de duración inferior a 3 meses.

Se comenzó realizando un muestreo probabilístico estratificado por unidades para reclutar, al menos, una unidad médica, una quirúrgica, una de cuidados críticos y una mixta. En las unidades que participaron en el proyecto de implantación se realizó un muestreo no probabilístico intencional que incluyera a todos los profesionales que cumplieran los criterios. En el caso de la unidad control, el muestreo fue no probabilístico consecutivo incluyendo a todos los profesionales de la unidad que decidieran participar una vez explicado el proyecto. El tamaño muestral venía condicionado por el número de profesionales que trabajan en las unidades participantes y que participaban en el proyecto de implantación.

Para la recogida de la información se creó y validó un cuestionario (índice de validez de contenido) que consta de 7 ítems más un conjunto de variables sociodemográficas y profesionales ${ }^{(17)}$. Atendiendo al objetivo de esta propuesta, se presenta el método de los tres ítems abordados.

El ítem 1 validado al castellano por Escobar Aguilar $\mathrm{G}$ et al, se ocupa de la utilización de la Investigación, según el ítem 6 del cuestionario de Estabrooks (18). Este ítem genera una red modo 2, caracterizadas por formarse con dos conjuntos de nodos, que permite explorar y generar la red social de utilización de las fuentes más habituales de información relacionada con la investigación. El ítem 2, siguiendo la metodología utilizada por Burt y Cross et al. ${ }^{(19)}$ explora redes sociales de ayuda y/o consejo para la transferencia de conocimiento. También resulta de él una red modo 2, que genera la red de ayuda y/o consejo para la transferencia de conocimiento. El ítem 7 se elaboró ad hoc para este estudio con el fin de conocer los lugares habituales donde se comparte la información relacionada con los resultados de la investigación. Al igual que en los ítems previos, se obtiene una red modo 2 para explorar lugares en los que se comparte información sobre investigación.

La participación se facilitó con un cuestionario online, con un envío inicial y otro recordatorio, para conseguir una mejor tasa de respuesta.

Tras concluir el periodo de participación, se trataron los datos recopilados, se aplicó el método ARS y se crearon los grafos con el software Pajek para visualizar las dinámicas del grupo de profesionales ${ }^{(20)}$. Cada red resultante se caracterizará con el inventario de nodos en cada grupo o modo, el número de relaciones entre ambos modos y la densidad de la red cuya medida permite conocer la proporción de las relaciones que ocurren frente el número de relaciones posibles. En una red de modo 2 el máximo de relaciones posibles resulta de multiplicar el número de nodos en cada conjunto. El valor que resulta de la densidad es un valor que oscila entre el 0 y el 1 , identificando el valor 0 un grafo desconectado y valor el 1 un grafo con relaciones completas. 


\section{RESULTADOS}

La participación de las seis unidades, Reanimación, UCl, Oncología, Ginecología, Paritorio y Psiquiatría (unidad control), ascendió a 77 profesionales, lo que supone una tasa global de participación del $71.96 \%$. El perfil sociodemográfico atiende a una participación de 9 hombres (11.7\%) y 68 mujeres (88.3\%) con una edad media de 42.9 (DE:11.48). Desde una perspectiva profesional 6 personas tienen una experiencia menor de 5 años (8\%), entre 6 y 10 años un total de 3 personas (4\%), entre 11 y 20 años 35 participantes (45\%), entre 21 y 30 años 23 de ellas $(30 \%)$ y, finalmente, 10 personas más de 30 años (13\%). El puesto ocupado se vincula a la asistencia en 73 personas (95\%) frente a 4 profesionales a la gestión (5\%).

La figura 1 es un grafo en el que se agrupan en clústers a los participantes ( 77 nodos en color gris) y las unidades con las que están vinculados laboralmente (6 nodos con diferentes colores). En la unidad de Reanimación se consiguió una tasa de respuesta del $97 \%$ (33 participantes o nodos), en UCl esa tasa fue del 100\% (8 nodos), en Oncología la tasa fue del 70\% (7 nodos), en Ginecología la tasa fue del $90 \%$ (9 nodos), en Paritorio la tasa fue del $34.8 \%$ (8 nodos) y en Psiquiatría la tasa fue del $54.5 \%$ (12 nodos).

Figura 1. Grafo de la red de unidades y participantes

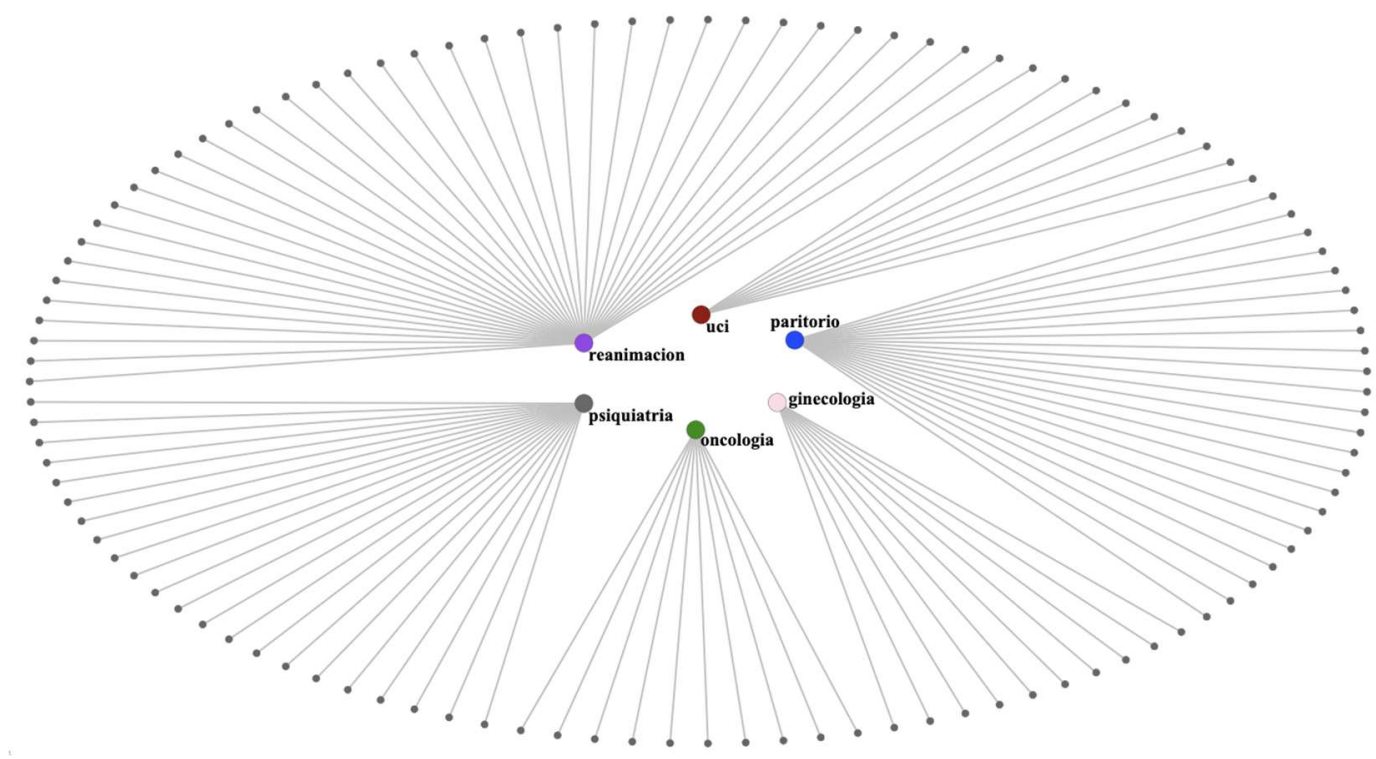

Fuentes de información para obtener resultados de investigación

El ítem 1 explora las fuentes de información que son utilizadas por los participantes para obtener información y/o consejo sobre resultados de investigación. La red que resulta, figura 2, está formada por dos conjuntos. En uno de ellos, los participantes (77 nodos en color gris); en el otro, las fuentes de información utilizadas: compañeros; bases de datos o artículos científicos (DBs); guías de práctica clínica o protocolos (Guías); Internet, redes sociales o webs (Internet); libros; sesiones, cursos o congresos (Sesiones) (6 nodos en color rojo). A su vez, también es una red 
ponderada. La ponderación es la importancia que cada participante asigna a cada fuente al decidir la frecuencia con la que la utiliza. Los diferentes tonos de gris asignados a los enlaces reflejan esa ponderación, a mayor intensidad de gris mayor frecuencia de uso.

Figura 2. Red de fuentes de información utilizada por los participantes. Elaboración propia con Pajek

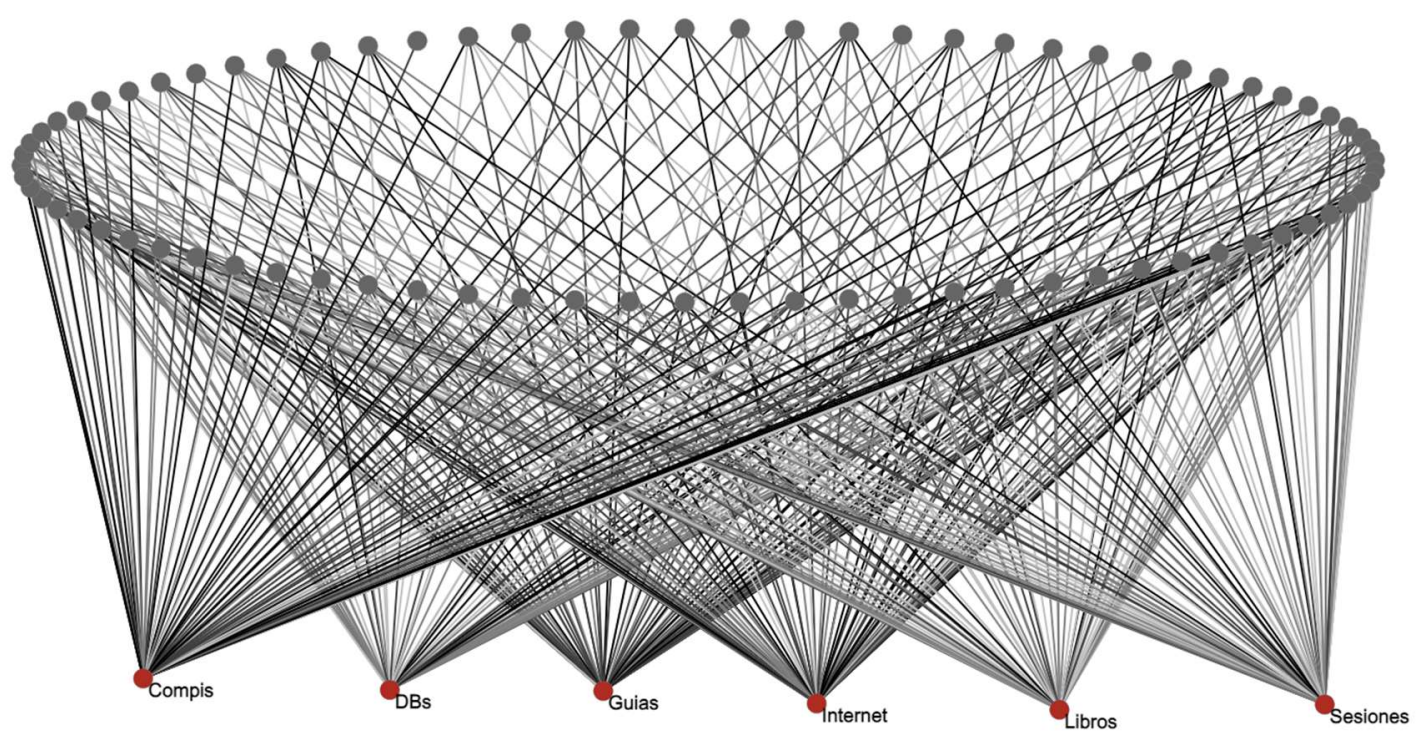

Las relaciones entre los nodos incluidos en ambos modos son direccionales, con el punto de inicio en los participantes y llegada a las fuentes. En esta red ocurrieron 424 relaciones; atendiendo a la frecuencia de utilización de la fuente (siendo 0 nunca, 1 raramente o no utilizada; 2 a veces, 3 frecuentemente, 4 o 5 siempre), permite reflejar que 38 relaciones tienen un valor igual a 0 , por lo tanto, fuentes nunca utilizadas; 88 relaciones tienen un valor de 1 , entonces fuentes utilizadas raramente; y el resto, 336, un valor superior a 1 , para el resto de usos.

La densidad de la red es 0.92 , un valor muy próximo a la unidad. Que eso ocurra es poco habitual en las redes de un único modo, pero en esta red ocurre como consecuencia de ser una pregunta planteada para que todos los participantes asignaran una frecuencia a cada fuente.

Los compañeros fue la fuente que más elecciones concentró y que mayor ponderación alcanza, mientras que la fuente que aglutina a las bases de datos bibliográficas y/o artículos científicos fue la menos seleccionada como fuente para informarse. La distribución de todas las fuentes consultadas con las respuestas (orden descendente) tanto absolutas como ponderadas y la puntuación media que resulta de dividir la ponderación ( $\Sigma$ frecuencia) entre el número de respuestas, se recoge en Tabla 1. 
Tabla 1. Fuentes de información consultadas: respuestas, respuestas ponderadas $\sum$ y puntuación media

\begin{tabular}{lccc}
\hline Fuentes consultadas & $\mathbf{N}$ respuestas & $\sum$ frecuencia & Puntuación media \\
Compañeros & 76 & 224 & 2.95 \\
BD_artículos & 63 & 133 & 2.11 \\
Guías-protocolos & 73 & 200 & 2.74 \\
Internet & 74 & 203 & 2.74 \\
Libros & 68 & 129 & 1.9 \\
Sesiones & 70 & 144 & 2.06 \\
\hline
\end{tabular}

Considerando las unidades de los participantes, tanto en Reanimación como en Psiquiatría (control) la fuente más utilizada fue los compañeros y la menos utilizada las bases de datos o artículos. En el resto de las unidades, UCl, Oncología, Paritorio y Ginecología, la fuente más utilizada fueron las guías de práctica clínica o protocolos y las menos utilizadas los libros, sesiones clínicas y bases de datos o artículos.

\section{Grupos profesionales como fuente de información o para consejo}

El ítem 2 explora los grupos de profesionales a los que se consulta para obtener información y/o consejo sobre resultados de investigación. Es un ítem donde, además de contar con opción múltiple y su valoración, los participantes cuentan con la posibilidad de añadir otras unidades a las que se vinculan los profesionales para obtener información y/o consejo.

De este ítem se obtiene la red incluida en la figura 3 con sus dos modos, donde uno incluye a los participantes (77 nodos en color gris) y el segundo a los profesionales de los que se obtiene información (10 en color rojo). El vínculo entre ambos modos es ponderado y se visualiza gracias a los diferentes tonos de gris asignados a los enlaces que reflejan esa ponderación, a mayor intensidad de gris mayor frecuencia de uso. La distribución de esos valores coincide con la expresada para el ítem 1. 
Figura 3. Red de grupos profesionales como fuente de información.

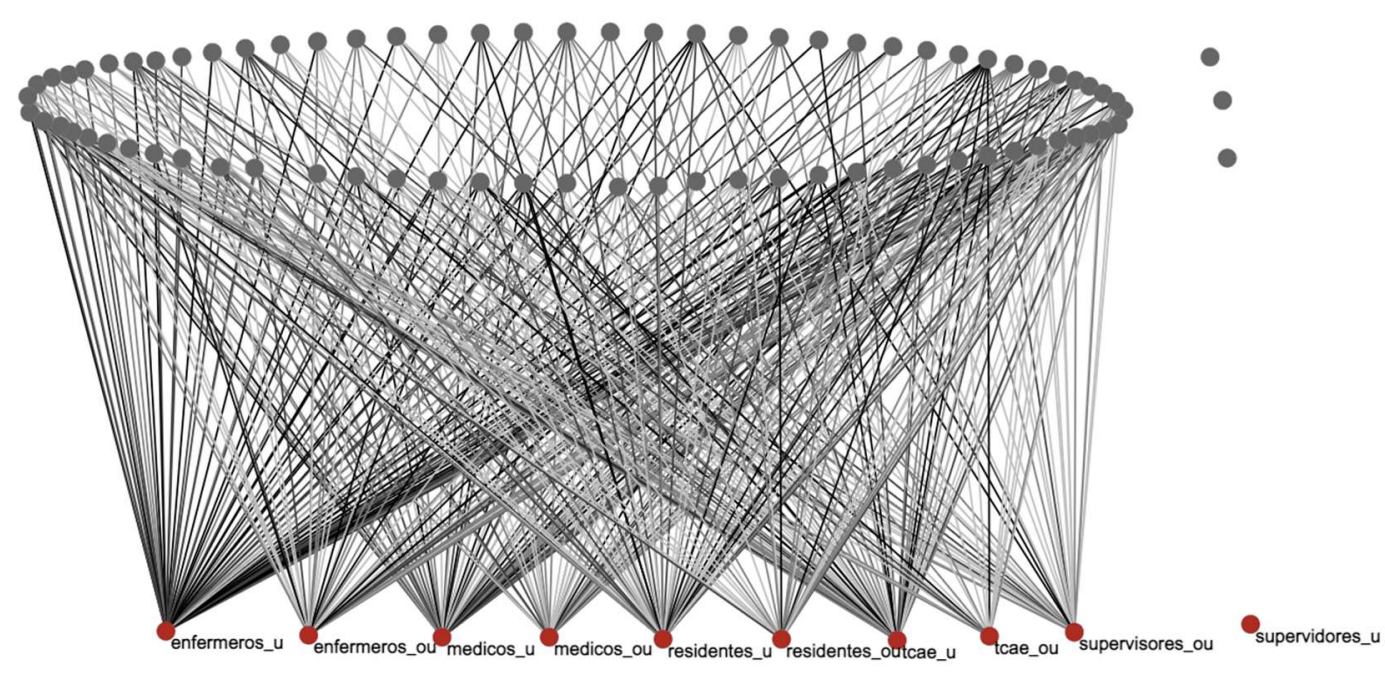

Elaboración propia con Pajek

Entre ambos modos ocurren 446 relaciones de las cuales 146 tienen un valor igual a 1 (que sería raramente) y la diferencia, 300 , un valor diferente a 1. En el último grupo mencionado se incluyen aquellas respuestas en las que los participantes no han respondido o los que indican nunca (en ambos casos 0 ).

La densidad de la red es 0.58 , y el valor confirma, que de entre todos los profesionales, en varias ocasiones no se valoró a muchos de ellos o eran profesionales de los que no se obtiene información o consejo.

Frente a ellos, los profesionales de los que se obtiene con más frecuencia información y que mayor ponderación alcanzan son los enfermeros de la misma unidad. Si bien, el profesional nunca considerado son los supervisores de la unidad (nodo del segundo modo aislado en color rojo). También aparecen aislados tres participantes, nodos grises, hecho que se puede interpretar como reflejo de que ningún grupo de profesionales sean para ellos una fuente de información. La distribución de todos los profesionales consultados con las respuestas tanto absolutas como ponderadas y la puntuación media que resulta de dividir la ponderación ( $\sum$ frecuencia) entre el número de respuestas, se recoge en Tabla 2.

Tabla 2. Profesionales consultados como fuente de información: respuestas, respuestas ponderadas $\Sigma$ y puntuación media

\begin{tabular}{lccc} 
Profesionales & $\begin{array}{c}\mathbf{N} \\
\text { respuestas }\end{array}$ & $\begin{array}{c}\sum \\
\text { frecuencia }\end{array}$ & $\begin{array}{c}\text { Puntuación } \\
\text { media }\end{array}$ \\
\hline Enfermeros unidad & 74 & 230 & 3.10 \\
Enfermeros otra unidad & 48 & 100 & 2.08 \\
Médicos unidad & 63 & 146 & 2.32 \\
Médicos otra unidad & 42 & 76 & 1.81 \\
Residentes unidad & 59 & 124 & 2.10
\end{tabular}


Residentes otra unidad

Supervisores unidad

Supervisores otra unidad

TCAE unidad

TCAE otra unidad
32

0

39

64

25

Cuando los participantes han dejado constancia de otras unidades a las que se vinculan para obtener información y/o consejo, para el perfil de médicos de otra unidad, fue la unidad de Medicina Interna (11) la más frecuente; cuando se indicó que se consultaban a otros profesionales que se ajustan al perfil de supervisores de otras unidades, es la supervisión de $\mathrm{UCI}$ la que mayor frecuencia acumula (13); cuando se optó por reflejar a qué otros profesionales se consultaba dentro del perfil de enfermeros de otras unidades, son los profesionales de $\mathrm{UCl}$ los más frecuentemente consultados (12); cuando se señaló que se consultaban a otros profesionales dentro del perfil TCAE de otras unidades, de nuevo los de $\mathrm{UCl}$ son los más frecuentes (6); cuando se mostró que se consultaban a otros profesionales dentro del perfil de residentes de otras unidades, son los de Paritorio los más frecuentes (6).

\section{Lugares donde se comparte información}

El ítem 7 explora los lugares o entornos en los que se ha compartido información relacionada con los resultados de investigación, limitado a los 12 últimos meses. Para este ítem se pide a los participantes que limiten su respuesta a 3 elecciones por orden de prioridad $\left(1^{\circ}, 2^{\circ} \circ 3^{\circ}\right)$.

De él se obtiene la red incluida en la figura 4 con sus dos modos, donde el modo un modo incluye a los participantes (77 nodos en color gris) y el segundo modo son los lugares donde se comparte ( 9 en color rojo). El uso de la escala de grises para las relaciones de esta red no se usa para las intensidades, ya que se expresarían en el sentido inverso. Quedarían menos intensas las primeras elecciones y más intensas las elecciones en tercer lugar. Por esa razón, todas se visualizan con la misma intensidad. 
Figura 4. Red de lugares donde se comparte información.

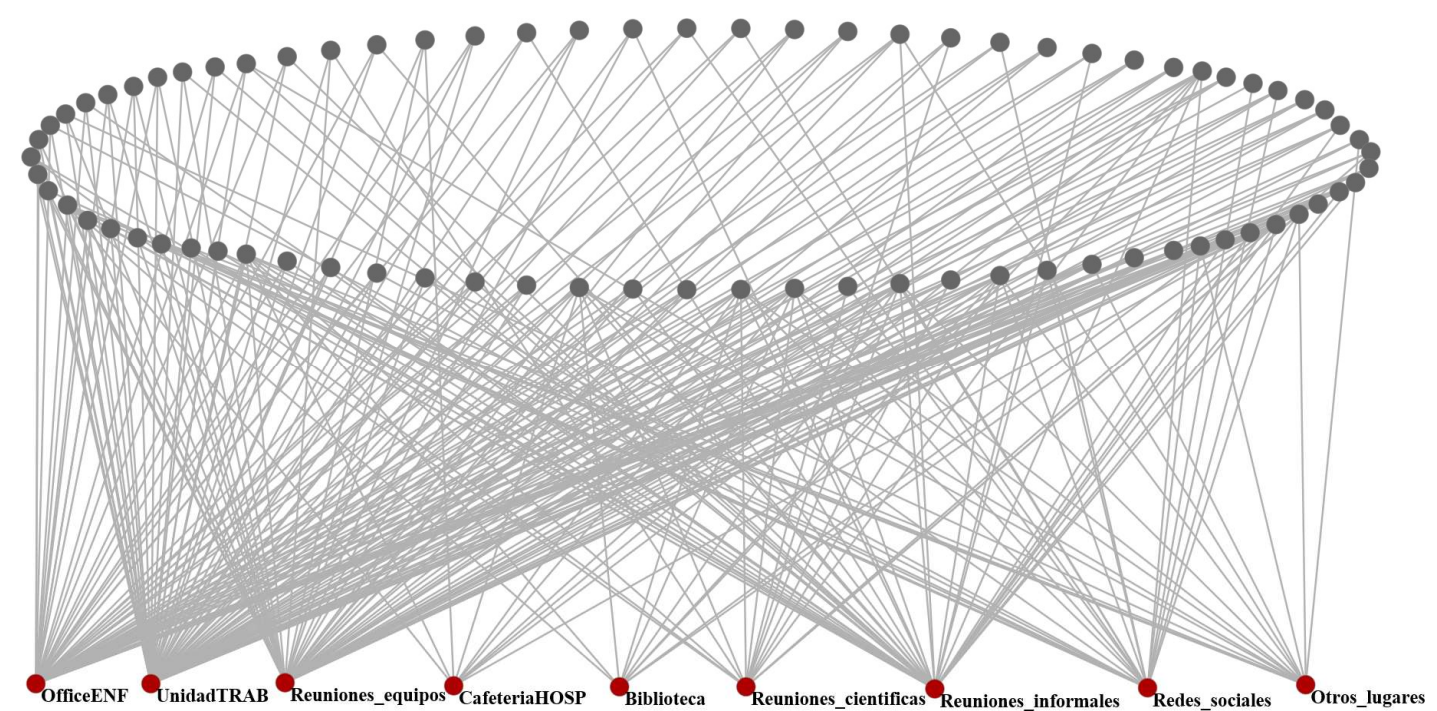

Elaboración propia con Pajek

Las relaciones entre los nodos incluidos en ambos modos son direccionales, con el punto de inicio en los participantes, considerando en esta ocasión que el número máximo de elecciones es 3 y que siempre como destino, se seleccionan los lugares en los que se comparte información. Ocurrieron 289 relaciones, de las que 105 tienen un valor igual a 1 (primer lugar) y 184 para las segundos y terceros lugares.

La densidad de la red es 0.42 , un valor esperado como consecuencia del enunciado de la pregunta, máximo tres elecciones de entre todos los posibles lugares o entornos en los que se ha compartido información relacionada con los resultados de investigación. Si bien el valor debiera estar próximo a 0.33 de densidad en esta red, por esas tres elecciones solicitadas de entre las nueve posibles. Pero algunos participantes asignaron una posición de 1, 2 o 3 simultáneamente a más de tres fuentes.

La unidad es el lugar más frecuentemente señalado como primera elección para compartir información, seguido del estar/office y como tercera, las reuniones de equipo. En la tabla 3, se incluye el patrón de elección de los participantes organizada por lugares.

Tabla 3. Lugares y elección donde se comparte información

$\begin{array}{lccc}\text { Lugares } & \mathbf{1}^{\mathbf{a}} \text { elección } & \mathbf{2}^{\mathbf{a}} \text { elección } & \mathbf{3}^{\mathbf{a}} \text { elección } \\ \text { Estar/Office } & 17 & 29 & 11 \\ \text { Dentro de la Unidad } & 50 & 15 & 6 \\ \text { Reuniones equipo } & 17 & 13 & 20 \\ \text { Cafetería } & 2 & 0 & 8 \\ \text { Biblioteca } & 2 & 2 & 6\end{array}$


Eventos/reuniones científicas

Reuniones informales

Redes sociales

Otros lugares fuera del

Hospital
4

4
4

11

10

2
10

18

3

10

Muchos participantes no le asignaron puesto a determinados entornos. Así ocurrió para biblioteca (67), cafetería del hospital (67), eventos-reuniones científicas (61), reuniones informales (41), reuniones de equipo (27) y estar/office de enfermería (20). Para otros lugares fuera del hospital donde se compartía información algunos participantes tampoco asignaron puesto (61); cuando hubo asignación, los lugares identificados fueron externos al ámbito hospitalario o casa en dos ocasiones; puntualmente, cafetería, consulta de nutrición propia, reunión informal, unidad docente de matronas, vestuario y otros hospitales (La Paz). Algo similar ocurre con las redes sociales (Whatsapp, Facebook) como lugar para compartir información, que también acumula como lugar una importante ausencia de asignación de puesto (54); que se completa con qué red social se utiliza y que en orden descendente es Whatsapp (8), Facebook (4), Instagram (2) y Twitter (1).

\section{DISCUSIÓN}

Considerando que la tasa de respuesta del estudio fue del $71,96 \%$, estudios con el método ARS obtuvieron tamaños muestrales similares al nuestro y una tasa de respuesta entre el $77-90 \%{ }^{(14,21)}$.

El ítem 1 que exploraba las fuentes de conocimiento utilizadas por los profesionales de enfermería para obtener información o consejo sobre resultados de investigación, mostró que en la red de este ítem los compañeros fue la fuente más elegida y con mayor ponderación; mientras que la fuente que aglutina a las bases de datos bibliográficas y/o artículos científicos fue la menos seleccionada como fuente para informarse. Esta red hace visible la hegemonía del conocimiento tácito reflejado por muchos autores en la literatura ${ }^{(2,22)}$. Es decir, el conocimiento experiencial sigue siendo una fuente importante para la resolución de problemas; lo que coincide con autores que reflejan que la experiencia se sitúa como recurso de información prioritario, siendo un elemento indispensable en la adquisición de conocimiento experto enfermero(23).

Sin embargo, si se analiza este ítem considerando el tipo de unidad surgen otros matices. De las 6 unidades analizadas, en 2 (Reanimación y Psiquiatría) la fuente más utilizada fueron los compañeros (conocimiento tácito o experiencial que puede estar sustentado en la investigación o no), pero en las cuatro unidades restantes ( $\mathrm{UCl}$, Oncología, Ginecología y Paritorio) consideraron las guías de práctica clínica y protocolos como la fuente de conocimiento más habitual. Esto puede estar relacionado con el grado de especialización de determinadas unidades en las que se trabaja con esas fuentes de información basadas, en muchos casos, en los resultados de la investigación. En esta línea también se está estudiando el impacto de la especialización (especialistas en formación) y de la práctica avanzada en el desarrollo de la práctica basada en la evidencia (PBE) y, por tanto, en la utilización de la 
investigación por los profesionales de enfermería. El nivel de experiencia implícito en estos nuevos roles se asocia a mayores aptitudes para sintetizar y aplicar la información derivada de la investigación ${ }^{(24)}$.

Por otro lado, se evidencia que los recursos electrónicos que permiten acceder a información científica de manera inmediata (bases de datos o artículos en acceso abierto), siguen siendo una fuente poco utilizada. En este estudio la edad media de la muestra está próxima a los 43 años, siendo probable la falta de formación relacionada con el acceso a recursos electrónicos y que ambos hechos puedan estar influyendo en la escasa elección de esta fuente. Esto coincide con estudios realizados al respecto, en los que la falta de conocimiento y habilidades en la búsqueda y evaluación de estudios de investigación publicados, supone una barrera para la utilización de la investigación ${ }^{(18,25)}$.

El ítem 2 exploraba los grupos de profesionales a los que se consulta para obtener información y/o consejo sobre resultados de investigación. Su red muestra cómo los profesionales de enfermería de la propia unidad se convierten en la principal fuente de información y/o consejo sobre resultados de investigación. Luego se perfilan redes homófilas (profesionales con estatus e intereses similares) y horizontales (entre personas con el mismo estatus). Estudios previos señalan que los profesionales de enfermería suelen manejarse en redes verticales (8); esto puede significar un cambio respecto a la importancia o prestigio otorgado al conocimiento procedente de otras figuras profesionales, como pueden ser los médicos, y se focalice en compañeros de la misma categoría profesionales ${ }^{(26)}$.

Las redes horizontales son más efectivas para difundir la influencia de los compañeros y apoyar tanto la construcción como la reformulación del conocimiento; las redes verticales son más efectivas para la aprobación de decisiones jerarquizadas. Esto se refuerza con el hecho de que los supervisores de la propia unidad (profesionales con estatus e intereses diferentes) no hayan sido elegidos, en ningún caso, como fuente de información; ya que las redes que se tienden a tejer entre profesionales de enfermería con enfermeros de otro estatus suelen ser verticales y relacionadas con decisiones basadas en la autoridad ${ }^{(8)}$. Sin embargo, la literatura refleja que los profesionales de enfermería con funciones de gestión reportan mejores puntuaciones en actitudes, conocimientos y habilidades hacia la $\mathrm{PBE}^{(27)}$.

La adopción de conocimiento científico por las personas es más probable si son homófilas, es decir, si tienen antecedentes socioeconómicos, educativos, profesionales y culturales similares. Los profesionales de la salud trabajan dentro de grupos de iguales que comparten valores junto a creencias comunes y el comportamiento individual puede verse fuertemente influenciado por estos elementos ${ }^{(7)}$.

El ítem 7 exploraba los lugares o entornos en los que se ha compartido información relacionada con los resultados de investigación, limitado a los 12 últimos meses. La unidad es el lugar más elegido para compartir información, como segunda elección el estar/office y como tercera elección las reuniones de equipo. Lo que evidencia que el ámbito laboral es considerado como el principal entorno para compartir información. Un estudio de ARS realizado en el contexto de un proyecto de implantación de evidencia, señala las reuniones de equipo como un contexto idóneo para intercambiar 
información; dado que la sobrecarga asistencial de las unidades impide compartir información de manera adecuada ${ }^{(28)}$.

Quizá la edad de la muestra pueda influir en la menor elección de lugares externos al ámbito laboral o el uso de las redes sociales donde compartir información relacionada con los resultados de investigación ${ }^{(29)}$.

Como líneas de trabajo futuras, es importante señalar que dentro del campo de la PBE los esfuerzos se están focalizando en generar evidencia sobre la efectividad de las intervenciones; sin embargo, las formas en las que la evidencia interactúa con la experiencia clínica y las redes profesionales deben ser abordados ${ }^{(3)}$. Por último, es importante señalar que contar con una unidad de control permitió perfilar si formar parte de un proyecto de implantación de evidencia puede producir el llamado "efecto contagio" descrito por algunos autores; efecto por el que la adopción del conocimiento se puede ver influenciado por la estructura de la red que lo va a adoptar, e incluye las características del conocimiento, de los actores y del contexto ${ }^{(30)}$. Bien es cierto que la unidad de control ha reflejado patrones diferentes en algún aspecto como puede ser las fuentes de conocimiento utilizadas por los profesionales para obtener información o consejo sobre resultados de investigación, pero se necesitarían abordajes estadísticos complementarios para poder analizar el efecto de la variable "tipo de unidad" en la elección de los diferentes profesionales como fuentes de información y los lugares donde se comparte.

\section{CONCLUSIONES}

Los profesionales de enfermería consideran el conocimiento experiencial de sus compañeros como la primera fuente para obtener información/consejo sobre resultados de investigación. Esa experiencia puede estar, o no, sustentada por la investigación. Esos profesionales crean redes homófilas-horizontales, por recurrir a profesionales con estatus e intereses similares, de los que obtienen información y/o consejo sobre resultados de investigación. Resaltar que los profesionales con puestos de gestión dentro de la unidad quedan excluidos como recurso para obtener esa información y/o consejo.

En unidades con cierto grado de especialización, los profesionales de enfermería utilizan guías de práctica clínica y protocolos como fuente principal de información. Mientras que las bases de datos y artículos científicos siguen siendo la fuente menos utilizada.

El entorno laboral físico con sus diferentes opciones (unidad, office o reuniones) es el más utilizado para compartir información sobre resultados de investigación.

\section{REFERENCIAS}

1. Ramírez A. La teoría del conocimiento en investigación científica: una visión actual. An la Fac Med. 2009;70(3):217-24.

2. Pérez-Fuillerat N, Solano-Ruiz MC, Amezcua M. Conocimiento tácito: características en la práctica enfermera. Gac Sanit [Internet]. 2019;33(2):191-6. Disponible en: http://dx.doi.org/10.1016/j.gaceta.2017.11.002

3. Rycroft-Malone J, Seers K, Titchen A, Harvey G, Kitson A, McCormack B. 
Nursing and Health Care Management and Policy. What counts as evidence in evidence-based practice? J Adv Nurs. 2004;47(1):81-90.

4. Nilsen $\mathrm{P}$, Ståhl $\mathrm{C}$, Roback $\mathrm{K}$, Cairney $\mathrm{P}$. Never the twain shall meet? - a comparison of implementation science and policy implementation research. Implement Sci. 2013;8(1).

5. Neta G, Brownson RC, Chambers DA. Opportunities for Epidemiologists in Implementation Science: A Primer. Am J Epidemiol. 2018;187(5):899-910.

6. Crisp N, Iro E. Comment Nursing Now campaign: raising the status of nurses. Lancet [Internet]. 2018;1-2. Disponible en: http://dx.doi.org/10.1016/S01406736(18)30494-X

7. Parchman ML, Scoglio CM, Schumm P. Understanding the implementation of evidence-based care: A structural network approach. Implement Sci [Internet]. 2011;6(1):14. Disponible en: http://www.implementationscience.com/content/6/1/14

8. Lau R, Stevenson F, Ong BN, Dziedzic K, Treweek S, Eldridge S, et al. Achieving change in primary care-causes of the evidence to practice gap: Systematic reviews of reviews. Implementation Science. 2016.

9. Dopson S. A view from organizational studies. Nurs Res. 2007;56(4):72-7.

10. Everett MG, Valente TW. Bridging, brokerage and betweenness. Soc Networks [Internet]. 2016;44:202-8.

Disponible

en:

http://dx.doi.org/10.1016/j.socnet.2015.09.001

11. Arias N, Calvo MD, Benítez-Andrades JA, Álvarez MJ, Alonso-Cortés B, Benavides C. Socioeconomic status in adolescents: A study of its relationship with overweight and obesity and influence on social network configuration. Int $\mathrm{J}$ Environ Res Public Health. 2018;15(9).

12. Quiroga E, Pinto-Carral A, García I, Molina AJ, Fernández-Villa T, Martín V. The influence of adolescents' social networks on alcohol consumption: A descriptive study of Spanish adolescents using social network analysis. Int J Environ Res Public Health. 2018;15(9).

13. Xu J, Yang R, Reblin M, Clayton M, Ellington L. Using Social Network Analysis to Investigate Positive EOL Communication. J Pain Symptom Manag. 2018;56(2):27380.

14. Creswick N, Westbrook Jl. Who Do Hospital Physicians and Nurses Go to for Advice about Medications? A Social Network Analysis and Examination of Prescribing Error Rates. J Patient Saf. 2015;11(3):152-9.

15. Maya I. 7 Usos Del Análisis De Redes En La Intervención Comunitaria. Rev Hisp para el Análisis Redes Soc [Internet]. 2016;27(2):1-10. Disponible en: http://revista-redes.rediris.es

16. Escobar-Aguilar G, Moreno-Casbas MT, González-María E, Martínez-Gimeno ML, Sánchez-Pablo C, Orts-Cortés I. The SUMAMOS EXCELENCIA Project. J Adv Nurs. 2019;(75):1575-84.

17. Martínez-Gimeno ML, Ovalle Perandones MA, Marqués-Sánchez $P$, Escobar-Aguilar G, Fernández-Martínez N, García-García E, et al. Validez de Contenido de un cuestionario para explorar redes sociales de transferencia del conocimiento. En: XXIII Encuentro Internacional de Investigación en Cuidados. Investen-Isciii, editor. Barcelona; 2019.

18. Escobar Aguilar G, Martínez-Gimeno M, MedinaTorres M, Martín de Castro E, Murillo Pérez M, Blanco Abril S. Patrones de utilización de la investigación por los profesionales de enfermería de los hospitales de Madrid. NURE Inv. 2015;12(79).

19. Burt RS. Structural holes: The social structure of competition. 1992.

20. De Nooy W, Mrvar A, Batagelj V. Exploratory Social Network Analysis with Pajek: Revised and Expanded Edition for Updated Software. Third Edition. Third Edit. 
Cambridge University Press, editor. New York; 2018.

21. Merrill JA, Yoon S, Larson E, Honig J, Reame N. Using social network analysis to examine collaborative relationships among $\mathrm{PhD}$ and DNP students and faculty in a research-intensive university school of nursing. Nurs Outlook [Internet]. 2013;61(2):109-16. Disponible en: http://dx.doi.org/10.1016/j.outlook.2012.08.001

22. Keith RE, Hopp FP, Subramanian U, Wiitala W, Lowery JC. Fidelity of implementation: development and testing of a measure. Implement Sci [Internet]. 2010;5(1):99. Disponible en: http://www.implementationscience.com/content/5/1/99

23. Greenhalgh T, Robert G, Macfarlane F, Bate P, Kyriakidou O. Diffusion of innovations in service organizations: Systematic review and recommendations. Vol. 82, Milbank Quarterly. 2004. p. 581-629.

24. Muñoz Jiménez D. From evidence-based nursing to healthcare practice: The evaluation of results as an integrating element. Enferm Clin. 2018;28(3):149-53.

25. Guadarrama-Ortega D. Barriers to the utilisation of research. Descriptive study performed on nurses at a hospital in the southwest of Madrid. Enferm Glob. 2016;(43):275-88.

26. Bond MO, Gaoue OG. Prestige and homophily predict network structure for social learning of medicinal plant knowledge. PLoS One [Internet]. 2020;15(10 October):1-27. Disponible en: http://dx.doi.org/10.1371/journal.pone.0239345

27. Ruzafa-Martínez M, López-lborra L, Armero Barranco D, Ramos-Morcillo AJ. Effectiveness of an evidence-based practice (EBP) course on the EBP competence of undergraduate nursing students: A quasi-experimental study. Nurse Educ Today [Internet]. 2015;38:82-7. Disponible en: http://dx.doi.org/10.1016/j.nedt.2015.12.012

28. Yousefi Nooraie R, Lohfeld L, Marin A, Hanneman R, Dobbins M. Informing the implementation of evidence-informed decision making interventions using a social network analysis perspective; a mixed-methods study. BMC Health Serv Res. 2017;17(1):1-14.

29. IAB SPAIN. Radiografía del usuario de Redes Sociales Español (estudio IAB Spain). 2017.

30. Fitzgerald L, Harvey G. Translational networks in healthcare? Evidence on the design and initiation of organizational networks for knowledge mobilization. Soc Sci Med [Internet]. 2015;138:192-200. Disponible en: http://dx.doi.org/10.1016/j.socscimed.2015.06.015

ISSN 1695-6141

(C) COPYRIGHT Servicio de Publicaciones - Universidad de Murcia 